\title{
Recent Trends in Digital Convergence Information System
}

\author{
Kyungyong Chung • Raouf Boutaba · Salim Hariri
}

Published online: 21 November 2014

CC Springer Science+Business Media New York 2014

Welcome to this special issue of the Wireless Personal Communications journal on recent trends in digital convergence information systems. The main goal behind the initiation of this special issue is to create a timely host be the vehicle for publishing selected research papers from academia and practitioners in different industries on this emerging topic. With the advancements in convergence technology, interactive multimedia communications, context awareness, and the proliferation of low cost compact sensing and computing devices, great attention is being paid to the study, development and deployment of digital convergence information systems in various sectors of the society such as healthcare, e-commerce, critical infrastructures, etc.. In healthcare, digital convergence information systems have the potential for lengthening life expectancy and most importantly improving Quality of Life (QoL). For these reasons and also to reduce healthcare costs (e.g., providing smarter healthcare to the elderly at home in an increasingly aging society), many governments worldwide, working with research centers and the industry, are to promoting the development of test projects for on- and off-line digital convergence-based health information systems. The industry has acknowledged their great potentials and is heavily investing in the development not only products that are enabling digital convergence information systems but also in the provisioning of digital convergence services for people as a business [1-3]. This special issue covers some of the hottest topics in digital convergence information systems, including: Personal

\footnotetext{
K. Chung $(\varangle)$

Department of Computer Information Engineering, Sangji University, 83 Sangjidae-gil, Wonju-si, Gangwon-do 220-702, Republic of Korea e-mail: kyungyong.chung@gmail.com

R. Boutaba

David R. Cheriton School of Computer Science, University of Waterloo, 200 University Avenue West, Waterloo, ON N2L 3G1, Canada e-mail: rboutaba@uwaterloo.ca

S. Hariri

NSF I/UCRC Center for Cloud and Autonomic Computing, University of Arizona, 1230 E. Speedway Blvd, Tucson, AZ 85721-0104, USA

e-mail: hariri@ece.arizona.edu
} 
computing for convergence systems; Personal and content protection; Ubiquitous computing for digital convergence; Software infrastructure, middleware and frameworks; Mobile computing for future communication systems; Agent technology in convergence information systems; Convergence system in smart health, telemedicine; Innovative applications of digital convergence information; Cooperative design in digital convergence environments; Artificial intelligence methodologies for convergence information. Specifically:

The paper by Ahn and Kim [4] introduces specific structures, like the lattice aspect ratio of windows, and discusses their value ranges, so the vortex air layer induced by natural convection flow prevents thermal loss of a room to the outside air, and consequently reducing energy consumption. The paper by Lee and Lee [5] introduces a step-node technique to provide a safe route for sensing data. To illustrate, the authors propose a safe route for ensuring the safety of firefighters. Performance evaluation is based on hop count for propagating sensing data from source to destination in the sensing network under normal and faulty processes.

The paper by Oh and Chung [6] improves speech detection using equivalent rectangular bandwidth (ERB) feature extraction. The features are extracted using an ERB filter bank cestrum, and a learning model following the acoustic one is constructed to improve speech recognition rate. The paper by Jo et al. [7] proposes an integrated smart education system. The latter is designed in the form of a structured plug-in for an integrated and expandable system, and can be customized according to user needs (government, enterprise, or school).

The paper by Ahn et al. [8] describes a toolkit for automating the evaluation of clinical decision support systems in the management of diabetes mellitus and hypertension in order to achieve speed, reliability, and freedom from human mistakes. The paper by Roh and Lee [9] investigates the variables that enable the measurement of attention and the development of tangible games to improve children attention. In particular, four variables are validated as usable to measure and assess attention through games and a $3 \mathrm{D}$ tangible game that improves children's attention level is developed.

The paper by Pattanamekar et al. [10] proposes an estimation approach for a commodity origin-destination (OD) matrix by using a sample commodity OD matrix from a commodity flow survey and a mode-specific OD matrices obtained from transportation records of freight carriers. The results show that the model is able to produce an acceptable commodity OD matrix, implying that the proposed approach is applicable to a real-world problem. The paper by Hong and Han [11] presents a secure and cost-efficient routing protocol for wireless sensor networks (WSNs) combining media access control (MAC) address-based authentication and a function to filter out low performance WSN members with low maintenance.

The paper by Li et al. [12] introduces an efficient method to leverage user interests and location information to support data dissemination in wireless ad hoc networks. Compared with existing methods, the proposed data dissemination reduces unnecessary data transmissions without requiring high network management overhead. The paper by Choi and Kang [13] describes a prototype automatic control system for vehicle sensors by applying a brain-computer interface (BCI) to a vehicle system for remote sensor control. The proposed BCI-based automatic control system can be applied to short-range transport models for assisting disabled users, and thereby improving their QOL.

The paper by Jeong et al. [14] introduces an attribute-based patient access control protocol to minimize violation of a patient's privacy using the patient's information saved in a smart phone in an emergency healthcare situation. The proposed protocol, to prevent the exposure of a patient's sensitive information to a third party, encodes the information with a signature key hashed by a random number created by the ubiquitous healthcare service center and the patient. The paper by Lee and Song [15] proposes a neural network using the cosine modulated 
symmetric exponential function, which is a non-monotonic function that can emulate spline networks by approximating polynomials and step functions.

The paper by Bae [16] proposes a security protocol that adheres to formal verification, including safety, deadlock, and livelock verifications, and uses the Casper Suite and Failures Divergence Refinement tools for validation. The paper by Kang et al. [17] introduces a real-time tracking and recognition system for interactive telemedicine health services using detection applied to posture recognition in telemedicine. Accordingly, a detected hand or finger can be used to implement a non-contact mouse, machine-to-machine (M2M) communication. This technology is valuable for controlling telemedicine health devices such as a pedometer health information reader, a glucose-monitoring device, or a blood pressure gauge.

The paper by Sodgerel et al. [18] presents a hand gesture recognition method using concentric coordinate information through image processing. The paper by Yang and Lee [19] proposes a method based on objective information to evaluate the impact on well-being as an indicator of QOL and which therefore can be utilized to promote the distribution of healthcare management resources.

The paper by Oh et al. [20] introduces a frequent-pattern network and a new method for discovering frequent patterns through the approximation of frequency counting on the network. The frequent-pattern network, where vertices and edges represent summarized information of transaction data, provides a user-centered environment based on the process of continuously mining frequent patterns. The paper by Wang et al. [21] introduces, analyzes, and compares the characteristics and important factors in adopting healthcare applications for present and potential customers. The authors argue that the results of this study can be used as baseline data for the development, supplementation, and operation of healthcare applications that reflect user requirements. The paper by Park et al. [22] proposes an almost blank subframe scheduling technique for interference mitigation under an M2M-based medical wireless body area network (WBAN). An implementation of an use case scenario with a medical WBAN and a distributed structure to recognize the effects on a WBAN device's processing volume is also provided.

This fine selection of papers has been achieved through a fruitful collaboration. We gratefully acknowledge the authors for their worthy contributions to this special issue. We would also like to thank all members of the ICDPM Technical Program Committee and the anonymous reviewers for their help in identifying original and innovative papers from the conference program and for their careful evaluation of earlier versions of the submitted papers. Accordingly, 19 high-quality papers were selected out of the 61 submitted resulting in a $31 \%$ acceptance rate. Last but not the least, we would like to thank Professor Ramjee Prasad, editor-in-chief of the international journal on Wireless Personal Communications, for his valuable remarks and his undeterred help throughout the publication process of this special issue.

\section{References}

1. Chung, K. Y. (2014). Recent trends on convergence and ubiquitous computing. Personal and Ubiquitous Computing, 18(6), 1291-1293.

2. Boutaba, R., Chung, K. Y., \& Gen, M. (2014). Recent trends in interactive multimedia computing for industry. Cluster Computing, 17(3), 723-726.

3. Oh, S. Y., Ghose, S., Jang, H. J., \& Chung, K. (2014). Recent trends in mobile communication systems. International Journal of Computer Virology and Hacking, 10(2), 67-70. 
4. Ahn, E., \& Kim, J. (2014). Windows and doors lattice structure for improving energy efficiency. Wireless Personal Communications. doi:10.1007/s11277-014-1766-3.

5. Lee, T. G., \& Lee, S. H. (2014). Safe sensing network system and evaluation for emergency information services. Wireless Personal Communications. doi:10.1007/s11277-013-1591-0.

6. Oh, S. Y., \& Chung, K. (2014). Improvement of speech detection using ERB feature extraction. Wireless Personal Communications. doi:10.1007/s11277-014-1752-9.

7. Jo, J., Park, G., Lee, D., \& Lim, H. (2014). An integrated teaching and learning assistance system meeting requirements for smart education. Wireless Personal Communications. doi:10.1007/s11277-014-1765-4.

8. Ahn, J. T., Park, G. H., Son, J., Lim, C. S., Kang, J., Cha, J., et al. (2014). Development of test toolkit of hard review to evaluate a random clinical decision support system for the management of chronic adult diseases. Wireless Personal Communications. doi:10.1007/s11277-014-1835-7.

9. Roh, C. H., \& Lee, W. B. (2014). A study of the attention measurement variables of a serious game as a treatment for ADHD. Wireless Personal Communications. doi:10.1007/s11277-014-1744-9.

10. Pattanamekar, P., Park, D., Lee, K. D., \& Kim, C. (2014). Genetic algorithm-based approach for estimating commodity OD matrix. Wireless Personal Communications. doi:10.1007/s11277-014-1808-x.

11. Hong, S., \& Han, K. H. (2014). Cost-efficient routing protocol (CERP) on wireless sensor networks. Wireless Personal Communications. doi:10.1007/s11277-014-1883-z.

12. Li, H., Bok, K., Chung, K., \& Yoo, J. (2014). An efficient data dissemination method over wireless ad-hoc networks. Wireless Personal Communications. doi:10.1007/s11277-014-1670-x.

13. Choi, S. J., \& Kang, B. G. (2014). Prototype design and implementation of an automatic control system based on a BCI. Wireless Personal Communications. doi:10.1007/s11277-014-1861-5.

14. Jeong, Y. S., Lee, S. H., \& Shin, S. S. (2014). Access control protocol based on privacy property of patient in m-healthcare emergency. Wireless Personal Communications. doi:10.1007/s11277-014-1767-2.

15. Lee, S. W., \& Song, H.S. (2014). Emulation of spline networks through approximation of polynomials and step function of neural networks with cosine modulated symmetric exponential function. Wireless Personal Communications. doi:10.1007/s11277-014-1664-8.

16. Bae, W. S. (2014). Formal verification of an RFID authentication protocol based on hash function and secret code. Wireless Personal Communications. doi:10.1007/s11277-014-1745-8.

17. Kang, S. K., Chung, K., \& Lee, J. H. (2014). Real-time tracking and recognition systems for interactive telemedicine health services. Wireless Personal Communications. doi:10.1007/s11277-014-1784-1.

18. Sodgerel, B., Lee, S. M., Kim, M. H., \& Yoo, H. Y. (2014). Circular hand gesture recognition algorithm using concentric circles. Wireless Personal Communications. doi:10.1007/s11277-014-1793-0.

19. Yang, J., \& Lee, Y. (2014). Development of measurement model for the value of QOL as an influential factor of metabolic syndrome. Wireless Personal Communications. doi:10.1007/s11277-014-1843-7.

20. Oh, K. J., Jung, J. G., \& Jo, G. S. (2014). Discovering frequent patterns by constructing frequent pattern network over data streams in E-marketplaces. Wireless Personal Communications. doi:10.1007/ s11277-014-1746-7.

21. Wang, B. R., Park, J. Y., Chung, K., \& Choi, I. Y. (2014). Influential factors of smart health users according to usage experience and intention to use. Wireless Personal Communications. doi:10.1007/ s11277-014-1769-0.

22. Park, R. C., Jung, H., \& Jo, S. M. (2014). ABS scheduling technique for interference mitigation of M2M based medical WBAN service. Wireless Personal Communications. doi:10.1007/s11277-014-2073-8.

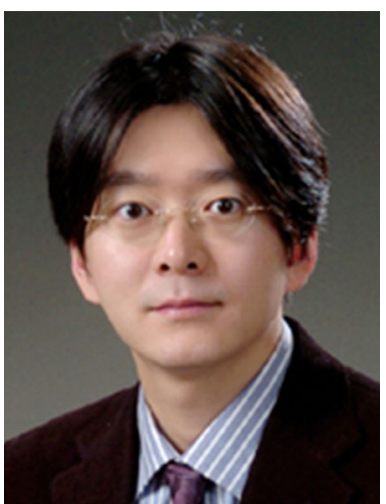

Kyungyong Chung is a professor in the Computer Information Engineering Department of Sangji University, Korea. He has worked for the Software Technology Leading Department of Korea IT Industry Promotion Agency (KIPA) in 2005. He received the B.S., M.S., and Ph.D. degrees in Computer and Information Engineering from Inha University in 2000, 2002, and 2005, respectively. His research interests are in Data Mining, Smart Health Services, Knowledge based Decision Support Systems, Intelligent Systems, Convergence, HCI, and Recommendation Systems. He served as General Co-Chair of the International Conference on Information Science and Application in 2013, General Co-Chair of 2nd International Conference on IT Convergence and Security in 2012, Executive Editing Director and in the Steering Committee of the International Conference on Convergence Technology, and Steering Committees member of the International Conference on Digital Policy \& Management. He is on editorial boards of several International Journals. 


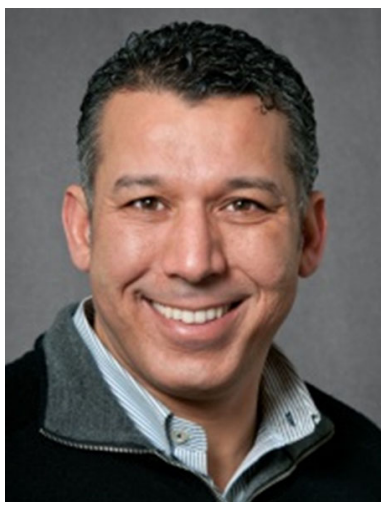

Raouf Boutaba received the M.Sc. and Ph.D. degrees in computer science from the University Pierre \& Marie Curie, Paris, in 1990 and 1994, respectively. He is currently a professor of computer science at the University of Waterloo (Canada). His research interests include resource and service management in networks and distributed systems. He served as a Distinguished Speaker for the IEEE Computer and the IEEE Communications Societies. He is the founding editor in chief of the IEEE Transactions on Network and Service Management (20072010) and on the editorial boards of several journals. He has received several best paper awards and other recognitions such as the Premiers Research Excellence Award, the IEEE Hal Sobol Award in 2007, the IEEE Fred W. Ellersick Prize in 2008, and the IEEE Joe LociCero and the Dan Stokesbury awards in 2009, the Salah Aidarous Award in 2012 and the McNaughton Gold Medal in 2014. He is a fellow of the IEEE and the Engineering Institute of Canada.

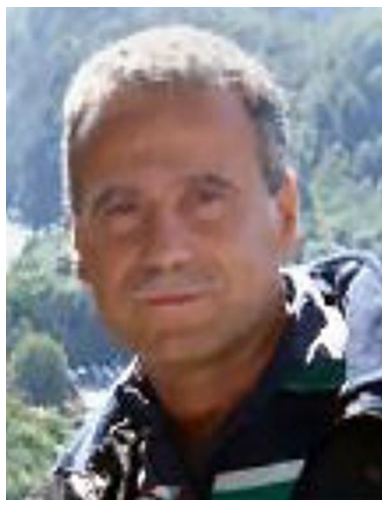

Salim Hariri is a Professor in the Department of Electrical and Computer Engineering at The University of Arizona. He received his Ph.D. in computer engineering from University of Southern California in 1986, and an MSc from The Ohio State University in 1982. He is internationally recognized as an authority on Autonomic Computing, autonomic network defense and protection, and High Performance Distributed Computing. He is the UA site director of NSF Cloud and Autonomic Computing (CAC) center and the Editor-In-Chief for the Cluster Computing Journal. He is coauthor / editor of four books on Autonomic computing, parallel and distributed computing: Autonomic Computing: Concepts, Infrastructure, and Applications (CRC Press, 2007), Tools and Environments for Parallel and Distributed Computing (Wiley, 2004), Virtual Computing: Concept, Design and Evaluation (Kluwer, 2001), and Active Middleware Services (Kluwer, 2000). Dr. Hariri is the founder of AVIRTEK: Autonomic Cyber Security, a startup and University of Arizona spinout company. Dr. Hariri has led the development of AVIRTEK's first commercial cyber security product, an "Automated and Integrated Management (AIM)" appliance that provides self-protection capabilities to all communications layers (Application Layer through the MAC layer). 